\title{
Numerical Research on Effects of Splitter Blades to the Influence of Pump as Turbine
}

\author{
Yang Sun-Sheng, ${ }^{1}$ Kong Fan-Yu, ${ }^{1}$ Fu Jian-Hui, ${ }^{2}$ and Xue Ling ${ }^{1}$ \\ ${ }^{1}$ Research Center of Fluid Machinery Engineering and Technology, Jiangsu University, Zhenjiang, Jiangsu 212013, China \\ ${ }^{2}$ Zhejiang Jiali Technology Co., Ltd, Hangzhou, Zhejiang 311241, China
}

Correspondence should be addressed to Yang Sun-Sheng, yangsunsheng@126.com

Received 27 September 2011; Accepted 3 December 2011

Academic Editor: Luis Gato

Copyright ( 2012 Yang Sun-Sheng et al. This is an open access article distributed under the Creative Commons Attribution License, which permits unrestricted use, distribution, and reproduction in any medium, provided the original work is properly cited.

\begin{abstract}
Centrifugal pumps can be operated in reverse as small hydropower recovery turbines and are cheaper than bespoke turbines due to their ease of manufacture. Splitter blades technique is one of the techniques used in flow field optimization and performance enhancement of rotating machinery. To understand the effects of splitter blades to the steady and unsteady influence of PAT, numerical research was performed. 3D Navier-Stokes solver CFX was used in the performance prediction and analysis of PAT's performance. Results show that splitter blades have a positive impact on PAT's performance. With the increase of splitter blades, its required pressure head is dropped and its efficiency is increased. Unsteady pressure field analysis and comparison show that the unsteady pressure field within PAT is improved when splitter blades are added to impeller flow passage. To verify the accuracy of numerical prediction methods, an open PAT test rig was built at Jiangsu University. The PAT was manufactured and tested. Comparison between experimental and numerical results shows that the discrepancy between numerical and experimental results is acceptable. CFD can be used in the performance prediction and optimization of PAT.
\end{abstract}

\section{Introduction}

Small hydroelectric power stations became attractive for generating electrical energy after the oil price crisis of the seventies. However cost per $\mathrm{kW}$ energy produced by these stations is higher than the hydroelectric power plants with large capacity. We considered the idea of using pumps as hydraulic turbines an attractive and important alternative. Pumps are relatively simple machine, easy to maintain, and readily available in most developing countries. From the economical point of view, it is often stated that pumps working as turbines in the range of 1 to $500 \mathrm{~kW}$ allow capital payback periods of two years or less which is considerably less than that of a conventional turbine [1].

Current research on PAT mainly focuses on the development of accurate prediction models for the turbine operation of different designs of centrifugal pumps [2-4]. However, the accuracy of these prediction models requires continuous verification and development. The optimization of PAT means generating more energy from current small hydropower resources. So some papers have been concentrated on the optimization of PAT [5-8]. Splitter blades technique is one of the techniques frequently used in turbomachinery design and application [9-11]. Thus, we consider the idea of adding splitter blades to PAT's impeller flow passage.

Because of the impulse of the blades that rotates with respect to the stator, the transmission of fluid machinery is unsteady [12]. The interaction of rotor and stator could result in the generation of pressure pulsations [13], component fatigue [14], and excessive noise and vibration [15]. Adding of splitter blades to impeller flow passage will cause an increase of impeller blade number and a change of the three-dimensional unsteady pressure flow field within PAT. Therefore, investigation into splitter blades to the influence of unsteady pressure field can be useful.

CFD has been widely used in the flow field visualization, optimization, and unsteady flow field analysis of rotating machinery $[16,17]$. Yet, investigation into unsteady pressure field of PAT using CFD is few [18]. In this paper, the all geometry of PAT including volute, impeller, and chambers 


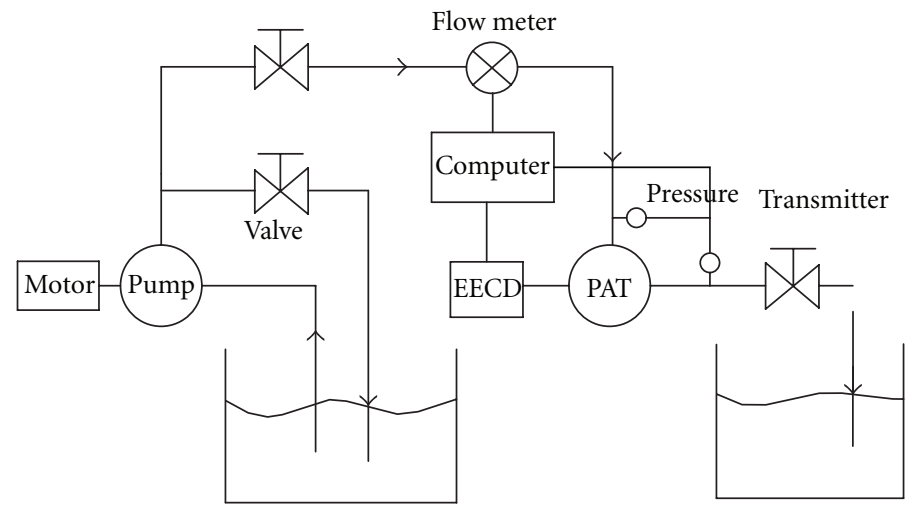

Figure 1: An open PAT test rig.

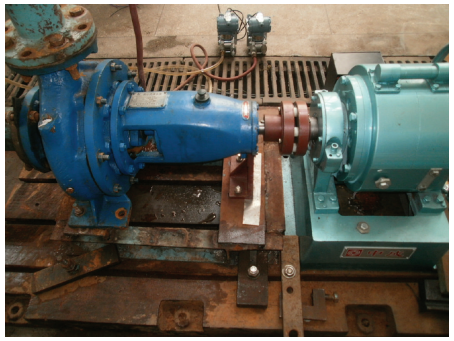

(a)

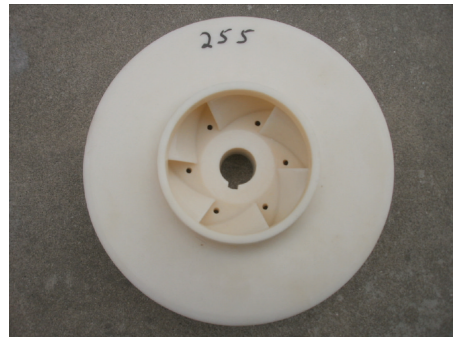

(b)

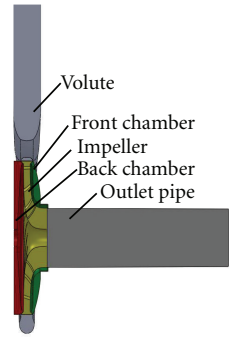

(c)

Figure 2: (a) Test PAT. (b) Impeller. (c) 3D model.

TABLE 1: Main geometric parameters of the designed PAT.

\begin{tabular}{lc}
\hline$b_{2}$ & 14.38 \\
\hline$D_{1}$ & 102 \\
$D_{2}$ & 255 \\
$D_{3}$ & 266 \\
$D_{4}$ & 65 \\
$D_{\mathrm{h}}$ & 30 \\
$z$ & $6 / 6+6$ \\
$e$ & 4 \\
$L_{\mathrm{sb}}$ & $0.8 L_{\mathrm{mb}}$ \\
$\beta_{1}$ & 19.5 \\
$\beta_{2}$ & 20 \\
$L$ & 15 \\
$\theta$ & 0.6 \\
$\phi$ & 131.5 \\
\hline
\end{tabular}

has been simulated, using commercial flow solver. The effects of splitter blades to the PAT's influence of steady performance and unsteady flow field are investigated.

A complete micro-hydropower test rig was established at Jiangsu University and was used for experimental verification of numerical results. The simulated PAT was made and tested using this test ring. All required parameters were measured for obtaining complete characteristic curves of the PAT. The accuracy of CFD results for steady state simulation was verified.

\section{Experimental Setup}

A complete laboratory model of PAT an open test rig, as shown in Figure 1, was set up at Jiangsu University. Highpressure fluid required for PAT energy recovery is supplied by a feed pump. An electric eddy current dynamometer (EECD) is installed to measure and consume energy generated by PAT and to regulate PAT's rotational speed. Its accuracy of torque measurement is $\pm 0.4 \% \mathrm{~N} \cdot \mathrm{m}$. The discharge is measured by a turbine flow meter with an uncertainty of $\pm 0.5 \%$. PAT inlet and outlet pressure are measured by pressure transmitter with an uncertainty of $\pm 0.4 \%$. After measuring all parameters, PAT required pressure head, shaft power, and efficiency were obtained.

\section{PAT Main Geometric Parameters}

The focus of the investigation is a single-stage centrifugal PAT with a rotational speed of $1500 \mathrm{rpm}$. Table 1 lists the main geometric parameters of the designed PAT. Figure 2(b) shows the tested PAT without splitter blades.

\section{Numerical Investigation}

ANSYS-CFX is a commercial 3D Navier-stokes CFD code that utilizes a finite-element-based finite-volume method to discrete the transport equations [19]. It is a fullyimplicit solver; thus it creates no time step limitation and is considered easy to implement. It is also a coupled solver 


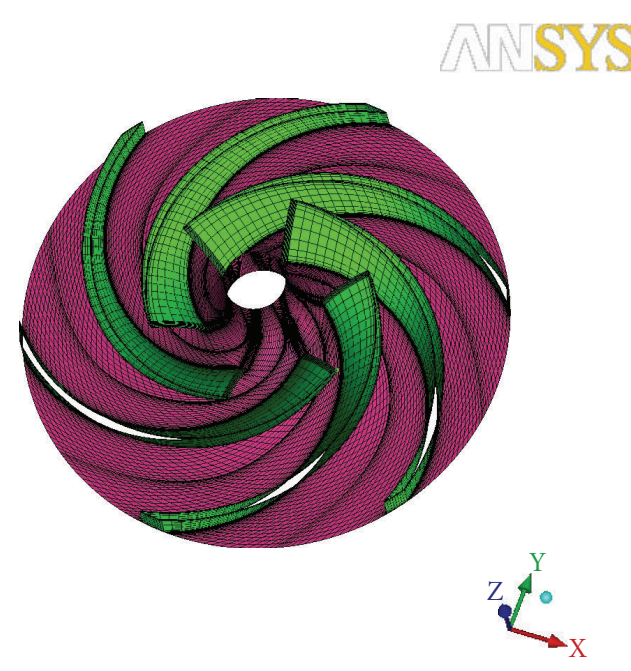

(a)
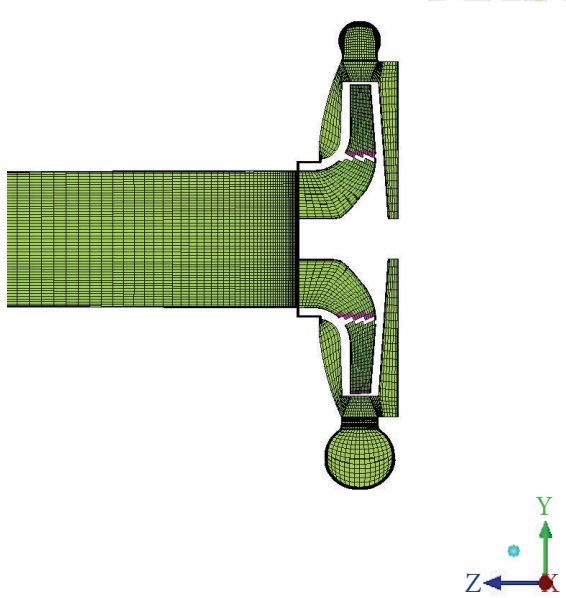

(b)

Figure 3: (a) Mesh of impeller. (b) Mesh assembly.
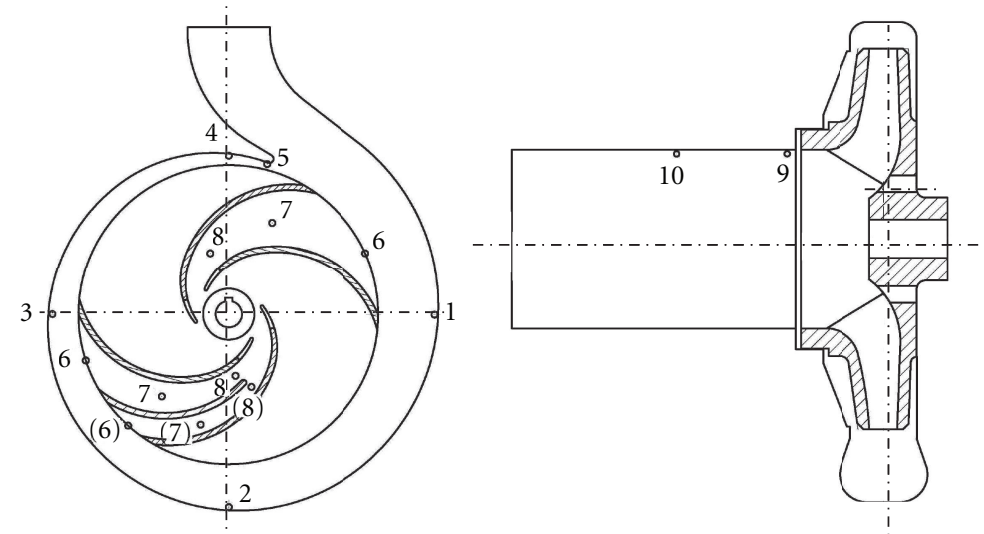

FIGURE 4: Location of pressure monitoring points.

TABLE 2: BEPs of experimental and numerical results.

\begin{tabular}{lcccc}
\hline Items & $Q\left(\mathrm{~m}^{3} / \mathrm{h}\right)$ & $\eta(\%)$ & $P(\mathrm{~kW})$ & $H(\mathrm{~m})$ \\
\hline Exp & 96.99 & 62.53 & 6.78 & 41.04 \\
Without splitter & 95.00 & 65.77 & 6.92 & 40.69 \\
With splitter & 90.00 & 69.19 & 6.24 & 36.80 \\
\hline
\end{tabular}

meaning that the momentum and continuity equations are solved simultaneously. This approach reduces the number of iterations required to obtain convergence and no pressure correction term is required to retain mass conversion, which leads into a more robust and accurate solver.

The fluid flow in a pump front and back chamber is usually neglected during CFD simulation. This simplification, which only has a minor effect on pump performance, is reasonable [20]. Unfortunately, the same is not true when considering in turbine mode. Due to the importance of PAT inlet flow, relatively large error has been reported in turbine mode [20]. In order to get a more accurate result, all fluid flow domain within the PAT control volume was modeled. The fluid flow domain was split into five component parts: volute, impeller passage, front and back chambers, and outlet pipe as indicated in Figure 2(c). This separation allowed each mesh to be generated individually and tailored to the flow requirements in that particular component. To get a relatively stable inlet and outlet flow, four times of the pipe diameter was extended in the PAT inlet and outlet section.

4.1. Mesh Generation. ICEM-CFD was used to generate structured hexahedral grid for each component part [19]. A grid-independent test of PAT's performance was performed; it was found that when mesh numbers were around 1 million, the variation of efficiency was within $0.5 \%$. The final number of mesh elements of volute, six blades impeller, front and back chambers, outlet pipe, and total number was 479710 , $325164,134956,129700,174720$, and 1244250, respectively. For impeller with splitter blades, the mesh number was almost the same. 


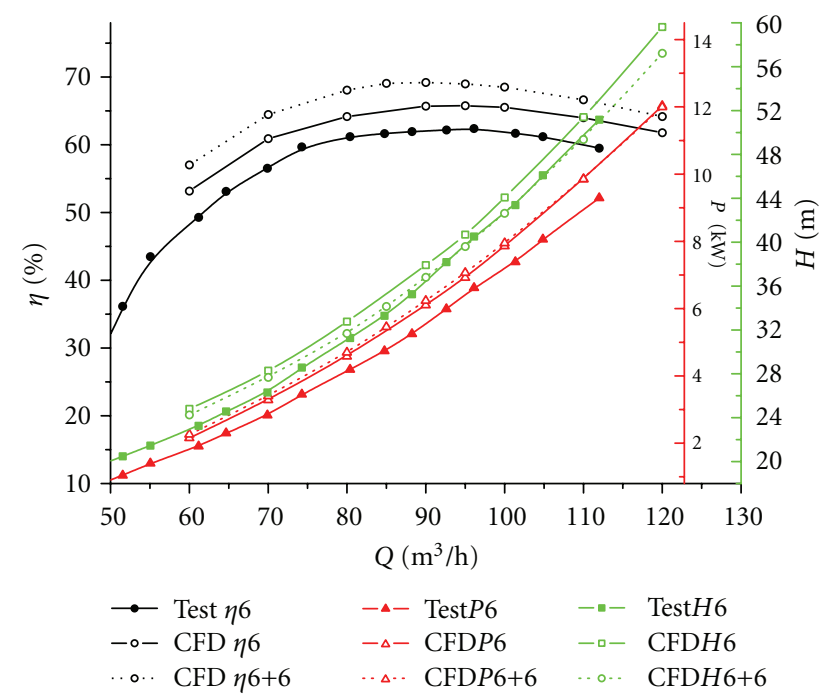

FIgURE 5: Comparison between experimental and numerical results.

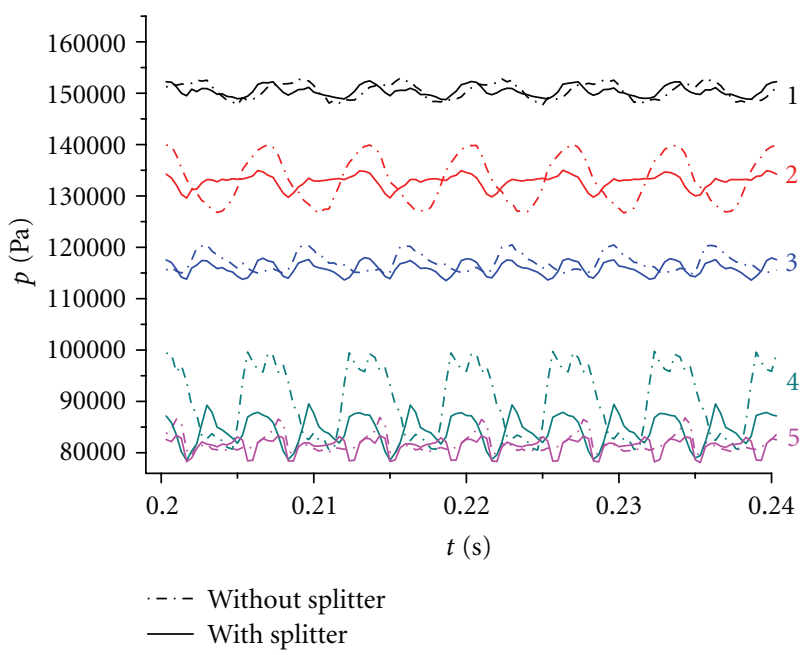

FIgURE 6: Time domain of pressure pulsation at volute monitoring points.

Care was taken in the PAT is outlet pipe mesh generation in order that mesh between the interface of outlet pipe and front chamber leakage is almost the same. The $y^{+}$near the boundary wall was around 40 . Due to the complexity of generating a structured mesh based on geometry, great efforts had been taken in the mesh generation of volute. Figure 3 gives a general view of the generated meshes.

4.2. Solution Parameters. The turbulence model selected was $\mathrm{k}-\varepsilon$ model. Standard wall function was used in the near wall treatment. The advection scheme was set to high resolution. The convergence criterion was $10^{-6}$. The fluid selected was water at $25^{\circ} \mathrm{C}$. All the wall surface roughness within the control volume was set to $50 \mu \mathrm{m}$. The inlet and outlet boundary conditions were set to static pressure inlet and mass flow rate outlet for both steady and unsteady simulation [19]. By changing the mass flow rate, performance curves of the PAT were acquired.
As the motion of the impeller blades relative to the stationary volute was central to the investigation, the analysis must involve multiple frames of reference. The volute and outlet pipe were set in stationary frame of reference and the impeller was set in rotary frame of reference. The interfaces between two stationary components, rotary and stationary components, were set to general grid and rotor stator interface, respectively.

Steady-state simulation was served as initial condition for unsteady simulation. The total time was set to $0.44 \mathrm{~s}$, and the time period set for each time step is $3.33 \times 10^{-4} \mathrm{~s}$. The time period of one cycle is $0.04 \mathrm{~s}$, so 11 rounds were simulated for the unsteady simulation. To get a relatively stable flow field, the final 6 rounds were selected for analysis. Figure 4 shows the position of monitoring points. The interfaces between two stationary components, rotary and stationary components, were set to general grid and transient rotor stator interface, respectively. 


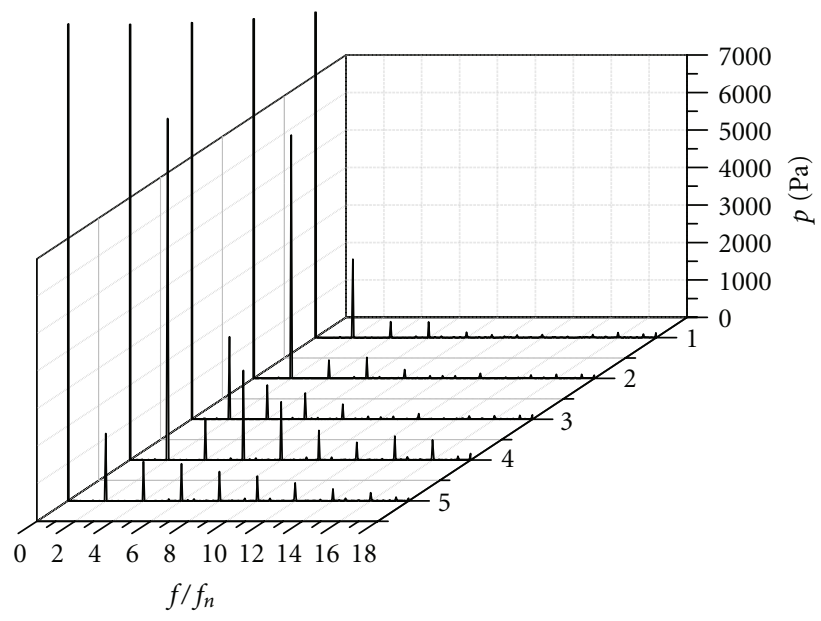

(a) Without splitter blades

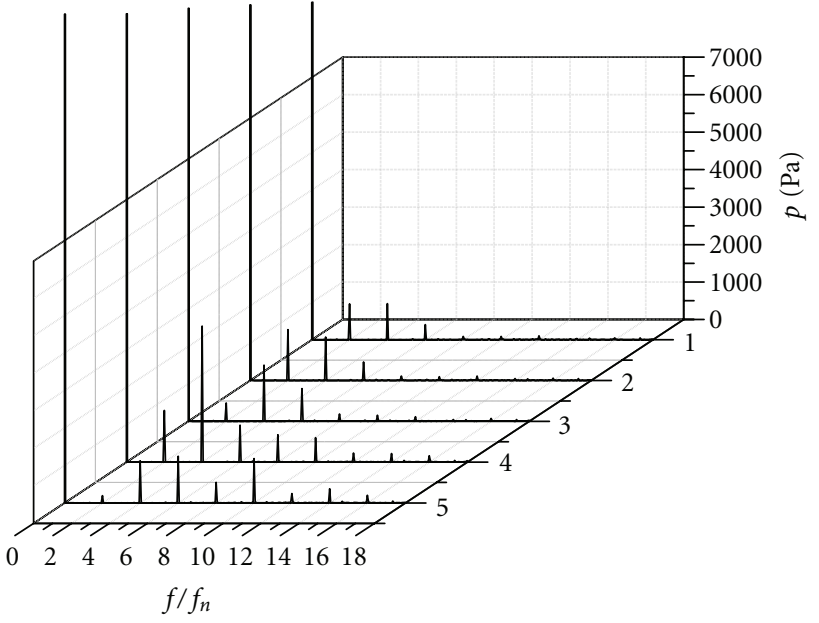

(b) With splitter blades

FIGURE 7: Frequency domain of pressure pulsation at volute monitoring points.

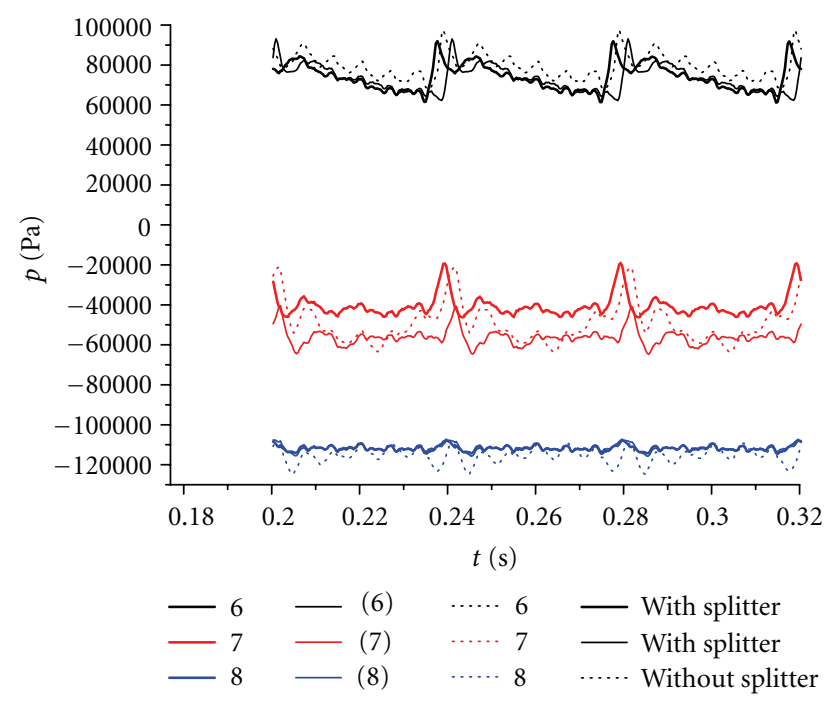

FIGURE 8: Time domain of pressure pulsation at impeller monitoring points.

\section{Results Analysis}

5.1. Performance Comparisons of Experimental and Numerical Results. An impeller with six blades was manufactured and tested. Numerical simulation of impeller with and without splitter blades was performed. Comparison of experimental and numerical results is presented in Figure 5. Table 2 lists its experimental and numerical results at the best efficiency points (BEPs). As is shown in Figure 5, the tendency of PAT's numerical predicted performance curves is in agreement with that of experimental. Numerical predicted efficiency, pressure head, and shaft power are higher than those of experimental. This overpredict of efficiency, pressure head, and shaft power may be attributed to the neglection of leakage loss through balancing holes and mechanical loss caused by mechanical seal and bearings. The comparison between experimental and steady-state numerical results indicates that the grid discretization and turbulence model are good for the PAT numerical simulation.

Numerical results comparison of PAT's impeller with and without splitter blades show that PAT's efficiency over the full operating range is increased when splitter blades are added to impeller flow passage. The required pressure head of PAT with splitter blades is decreased compared with that of without splitter blades. The variation of shaft power with and without splitter blades is minor.

5.2. Unsteady Pressure Field Comparison. The interaction of rotating impeller and stationery volute could cause strong pressure pulsation. To analyze PAT's unsteady pressure field and compare the pressure difference caused by the increase of splitter blade to PAT's impeller, unsteady pressure field analysis and comparison at a flow rate of $90 \mathrm{~m}^{3} / \mathrm{h}$ are presented. 
TABLE 3: Amplitude of frequency domain and MPF at volute monitoring points (see Figures 7(a) and 7(b)).

\begin{tabular}{|c|c|c|c|c|c|c|}
\hline Items & Frequency & 1 & 2 & 3 & 4 & 5 \\
\hline \multirow{4}{*}{ Without splitter } & $50 \mathrm{~Hz}$ & 2101.52 & 6497.02 & 2194.44 & 9119.44 & 1797.85 \\
\hline & $100 \mathrm{~Hz}$ & 425.34 & 491.59 & 911.75 & 1111.94 & 1051.60 \\
\hline & $150 \mathrm{~Hz}$ & 420.67 & 560.64 & 699.09 & 2390.07 & 987.26 \\
\hline & MPF & 1.44 & 3.73 & 1.66 & 5.24 & 2.03 \\
\hline \multirow{4}{*}{ With splitter } & $50 \mathrm{~Hz}$ & 956.43 & 1361.59 & 492.59 & 1379.13 & 190.40 \\
\hline & $100 \mathrm{~Hz}$ & 963.56 & 1160.57 & 1495.59 & 3628.93 & 1120.18 \\
\hline & $150 \mathrm{~Hz}$ & 400.03 & 498.83 & 870.31 & 984.72 & 1249.82 \\
\hline & MPF & 1.02 & 1.55 & 1.25 & 3.09 & 1.48 \\
\hline
\end{tabular}

TABle 4: Amplitude of frequency domain and MPF at impeller monitoring points (see Figures 9(a), 9(b), and 9(c)).

\begin{tabular}{|c|c|c|c|c|}
\hline Items & Frequency & $6 /(6)$ & $7 /(7)$ & $8 /(8)$ \\
\hline \multirow{5}{*}{ Without splitter (a) } & $8.33 \mathrm{~Hz}$ & 6018.02 & 11049.18 & 2256.43 \\
\hline & $16.67 \mathrm{~Hz}$ & 1649.96 & 2377.97 & 281.91 \\
\hline & $33.33 \mathrm{~Hz}$ & 2255.83 & 4226.27 & 1600.46 \\
\hline & $41.67 \mathrm{~Hz}$ & 2872.05 & 3407.42 & 1617.94 \\
\hline & MPF & 8.05 & 11.47 & 4.34 \\
\hline \multirow{5}{*}{ With splitter (b) } & $8.33 \mathrm{~Hz}$ & 7445.42 & 3633.10 & 164.20 \\
\hline & $16.77 \mathrm{~Hz}$ & 2417.61 & 2764.29 & 645.44 \\
\hline & $33.33 \mathrm{~Hz}$ & 2466.57 & 3954.63 & 1000.15 \\
\hline & $41.67 \mathrm{~Hz}$ & 2674.59 & 2972.82 & 458.17 \\
\hline & MPF & 8.55 & 7.58 & 2.02 \\
\hline \multirow{5}{*}{ With splitter (c) } & $8.33 \mathrm{~Hz}$ & 7195.57 & 1958.94 & 424.43 \\
\hline & $16.77 \mathrm{~Hz}$ & 2589.07 & 909.47 & 756.64 \\
\hline & $33.33 \mathrm{~Hz}$ & 2413.62 & 3585.52 & 1085.72 \\
\hline & $41.67 \mathrm{~Hz}$ & 2313.00 & 2206.07 & 617.10 \\
\hline & MPF & 8.59 & 6.75 & 2.30 \\
\hline
\end{tabular}

5.2.1. Volute. The absolute pressure pulsation comparisons with and without splitter blades within volute are plotted in Figure 6. As is shown in Figure 6, the absolute pressure gradually decreases along the volute flow channel. The maximum pressure pulsation among the five monitoring points is point 4 , which is about $20^{\circ}$ before the volute tongue. When splitter blades are added to the impeller, there is a significant drop of pressure fluctuation of the five monitoring points, especially at pressure monitoring points of 2 and 4 .

Figure 7 presents the frequency domain of PAT's absolute pressure pulsation after Fast Fourier Transform (FFT) with and without splitter blades. Table 3 lists the amplitude value at different frequencies. The maximum pressure fluctuation (MPF) is calculated from

$$
\mathrm{MPF}=\frac{p_{\max }-p_{\min }}{\rho g H} \times 100,
$$

where $p_{\max }$ and $p_{\min }$ are the maximum and minimal pressure; $\rho$ is the density of fluid; $H$ is PAT's required pressure head.

Figure 7(a) shows that the main frequency of PAT's without splitter blades is $50 \mathrm{~Hz}$. PAT's rotational speed is
TABLE 5: Amplitude of frequency domain and MPF at outlet pipe monitoring points (see Figure 11).

\begin{tabular}{lccc}
\hline Points & Frequency & 9 & 10 \\
\hline \multirow{3}{*}{ Without splitter } & $50 \mathrm{~Hz}$ & 3646.66 & 3112.41 \\
& $100 \mathrm{~Hz}$ & 523.45 & 705.60 \\
& $150 \mathrm{~Hz}$ & 385.90 & 383.59 \\
& $\mathrm{MPF}$ & 2.48 & 2.25 \\
\hline \multirow{3}{*}{ With splitter } & $50 \mathrm{~Hz}$ & 745.41 & 1416.07 \\
& $100 \mathrm{~Hz}$ & 754.98 & 524.37 \\
& $150 \mathrm{~Hz}$ & 544.05 & 510.22 \\
& $\mathrm{MPF} \mathrm{Hz}$ & 1.02 & 1.30 \\
\hline
\end{tabular}

$1500 \mathrm{rpm}$. The impeller rotational frequency $f_{n}$ is $25 \mathrm{~Hz}$. So PAT's main frequency of impeller without splitter blades is 2 times of the impeller rotational frequency. Also the highfrequency component of the pressure pulsation gradually increases as the flow channel is near the volute cutwater. Figure 7(b) indicates that when splitter blades are added to the impeller, its main frequency changes to $100 \mathrm{~Hz}$. Also the value of pressure amplitude is greatly dropped. 


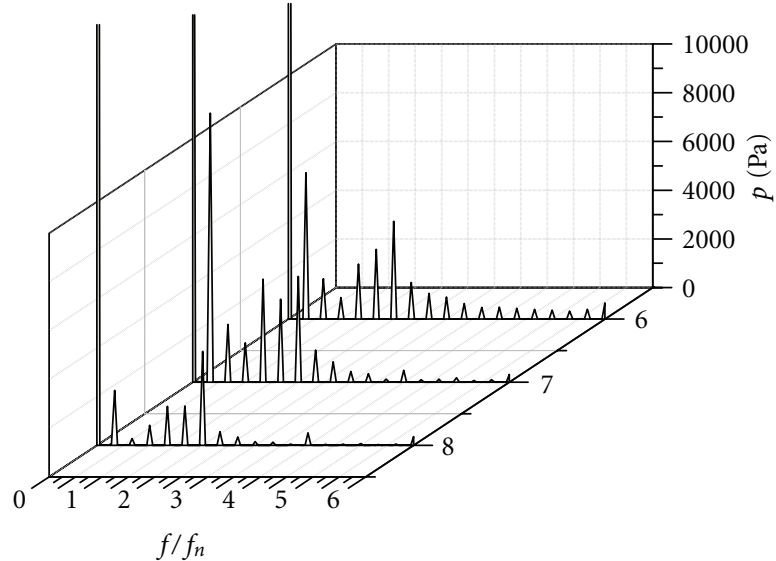

(a) Without splitter blades.

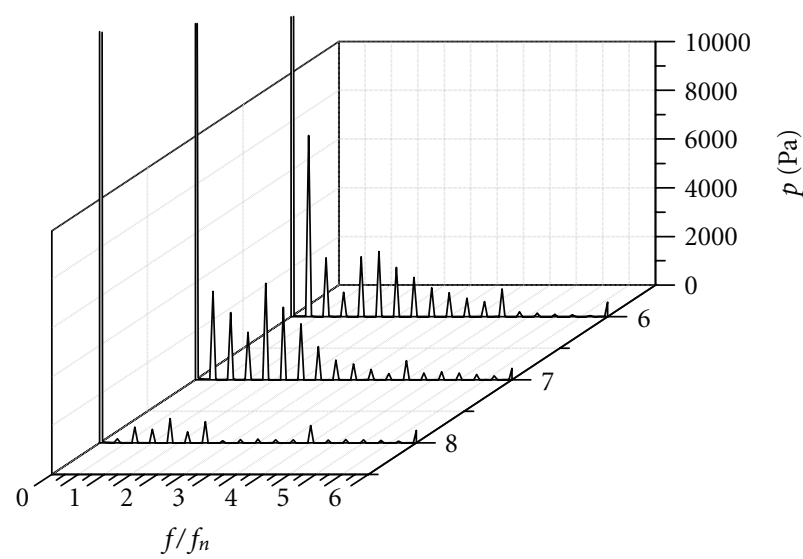

(b) With splitter blades

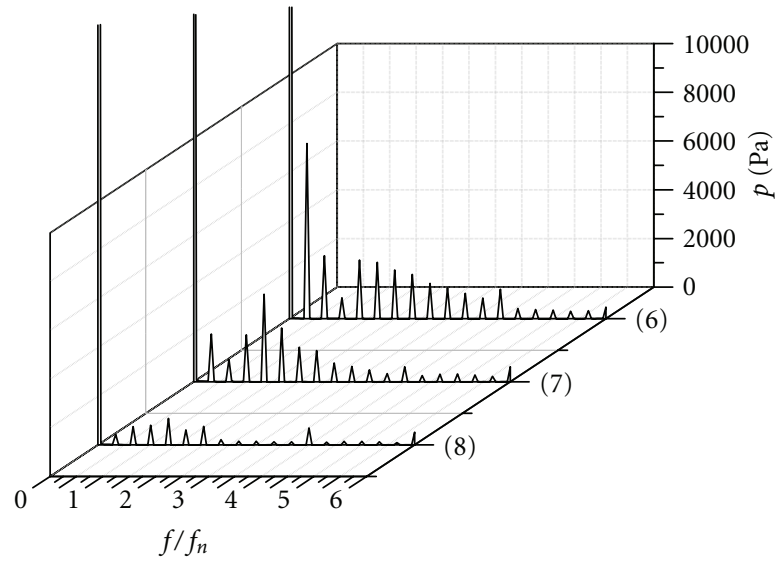

(c) With splitter blades

FIGURE 9: Frequency domain of pressure pulsation at impeller monitoring points.

Of the five investigated monitoring points, the minimum and maximum decreases of MPF are $24.70 \%$ and $58.44 \%$, respectively.

5.2.2. Impeller. The absolute pressure comparisons within impeller are plotted in Figure 8. Figure 9 presents the frequency domain of the monitoring points after FFT. Table 4 lists the amplitude value at different frequencies.

As is shown in Figure 8, there is only one peak value within one impeller rotational period. This is different from that of volute. The absolute pressure decreases dramatically along the impeller flow passage. This great drop of absolute pressure is due to the energy transfer of high-pressure energy to PAT's shaft power.

Figure 9 and Table 4 indicate that the main frequency of pressure fluctuation within impeller is $8.33 \mathrm{~Hz}$. So the blade passing frequency is $4.17 \mathrm{~Hz}$. Thus, we could see that the main frequency of pressure fluctuation within impeller is 2 times of the blade passing frequency.

Figures 8, 9 and Table 4 demonstrate that the maximum amplitude value of pressure fluctuation among the three monitoring points for impeller without splitter blades is point 7 . When splitter blades are added to the impeller, the amplitude value of pressure fluctuation at point 7 is decreased. And this decrease of pressure fluctuation also happens at pressure monitoring point 8 . The pressure fluctuation at impeller inlet area is almost the same for impeller with and without splitter blades. Also, it is observed that the amplitude of pressure fluctuation within two flow passages caused by the increase of splitter blades to impeller passage is almost the same.

5.2.3. Outlet Pipe. The variations of absolute pressure at monitoring points 9 and 10 within outlet pipe are plotted in Figure 10. Figure 11 shows the frequency domain of the pressure pulsation after FFT. Table 5 lists the amplitude value of pressure pulsation at different frequencies.

Figure 10 indicates that there are six peak values within one impeller rotational period. This is the same as that of volute. Figure 11 and Table 5 indicate that the main frequency of pressure fluctuation within outlet pipe is $50 \mathrm{~Hz}$. So PAT's main frequency is 2 times of the impeller rotational frequency. Also seen from Figures 10, 11 and Table 5, when splitter blades are added to the impeller, the amplitude of pressure fluctuation is decreased. And the MPF at monitoring points 9 and 10 is decreased by $58.87 \%$ and $42.22 \%$, respectively. 


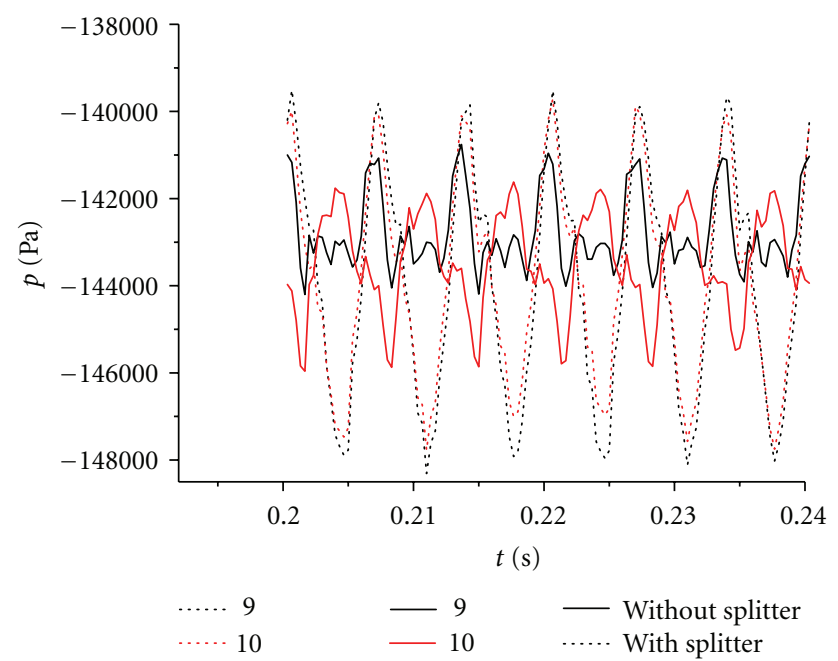

FIgURE 10: Time domain of pressure pulsation at outlet pipe monitoring points.

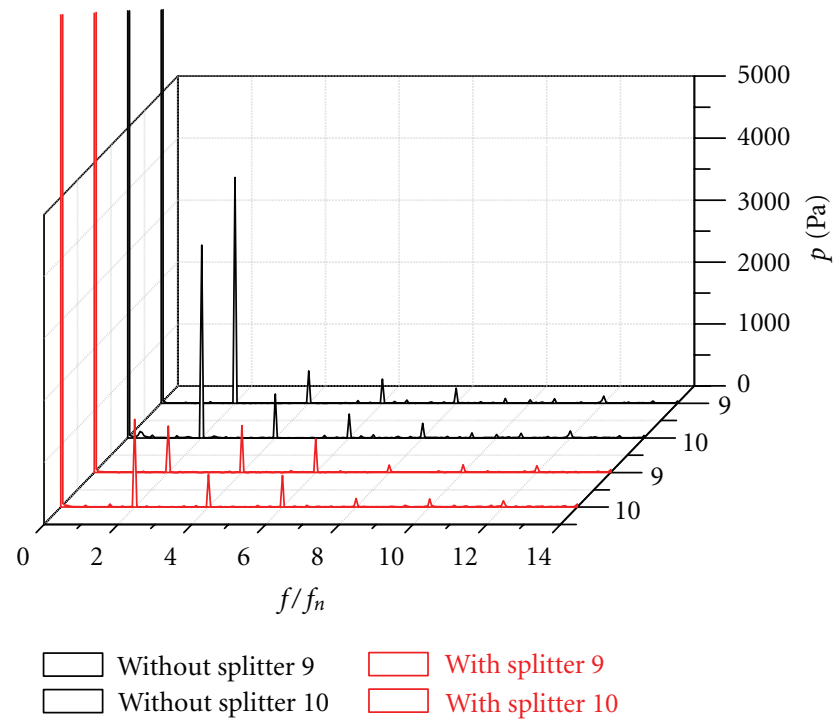

FigURE 11: Frequency domain of pressure pulsation at outlet pipe monitoring points.

\section{Conclusions}

Numerical investigation into splitter blade to the performance influence of pump as turbine was carried out.

An open PAT test rig was built. Experimental and numerical research on a single-stage PAT of impeller without splitter blades was carried out. In the process of numerical simulation, all domains except the leakage through the balancing holes and mechanical seals are included. Comparison between experimental and numerical results shows that CFD results are in good agreement with those of experimental and can be used in the performance prediction and optimization of PAT. CFD could be used in the optimization and analysis of pump as turbine.

Numerical predicted performance comparison of impeller with and without splitter blades shows that PAT's efficiency is increased and its required pressure head is decreased when splitter blades are added to impeller flow passage.
Investigation into unsteady pressure field within volute shows that the absolute pressure gradually decreases along the volute flow channel. The maximum amplitude value of pressure pulsation within volute is located before the volute tongue. When splitter blades are added to the impeller, there is a significant decrease of pressure fluctuation.

Due to the energy transfer of high-pressure energy to PAT's shaft power, the absolute pressure within PAT's impeller decreases dramatically along impeller flow passage. For impeller without splitter blades, the maximum value of pressure pulsation within impeller happens in the middle of impeller. When splitter blades are added, its amplitude of pressure pulsation is greatly decreased. Also it is observed that the pressure pulsation within the two flow passages caused by the increase of splitter blade to impeller flow channel is almost the same.

Analysis of pressure pulsation within PAT's outlet pipe shows that the absolute pressure gradually decreases along 
the outlet pipe. When splitter blades are added to the impeller, the amplitude of pressure fluctuation is also decreased.

\section{Nomenclature}

$b_{2}$ : Impeller inlet width, $\mathrm{mm}$

$D_{1}$ : Impeller outlet diameter, $\mathrm{mm}$

$\mathrm{D}_{2}$ : Impeller inlet diameter, $\mathrm{mm}$

$D_{3}$ : Volute base circle diameter, $\mathrm{mm}$

$D_{4}$ : Volute inlet diameter, $\mathrm{mm}$

$D_{\mathrm{h}}$ : Impeller hub diameter, $\mathrm{mm}$

$e$ : Impeller blade thickness, mm

$f_{n}$ : Impeller rotational frequency, $\mathrm{Hz}$

$H$ : Head, m

$L$ : $\quad$ Length of impeller wear ring, $\mathrm{mm}$

$L_{\mathrm{mb}}$ : Length of main blade, $\mathrm{mm}$

$L_{\mathrm{sb}}$ : Length of splitter blade, $\mathrm{mm}$

$n$ : $\quad$ Rotational speed, rpm

P: $\quad$ Power, W, kW

Q: Discharge, $\mathrm{m}^{3} / \mathrm{h}$

$y^{+}$: y plus

$z$ : Number of blades.

\section{Greek Symbols}

$\eta$ : Efficiency

$\theta$ : Splitter blade offset angle

$\phi$ : Blade wrap angle.

\section{Acknowledgments}

Special thanks are given to the national project entitled The Exploitation and Utilization of Hydraulic Power Recovery Turbines (2009GJC10007) and Doctoral innovation foundation of Jiangsu University entitled Research on High Pressure Fluid Power Recovery Device of Pump as Turbine (CX10B_012X).

\section{References}

[1] J. M. Chapallaz, P. Eichenberger, and G. Fischer, Manual on Pumps Used as Turbines, Vieweg, Braunschweig, Germany, 1992.

[2] M. Amelio and S. Barbarelli, "A one-dimensional numerical model for calculating the efficiency of pumps as turbines for implementation in micro-hydro power plants," in Proceedings of the 7th Biennial ASME conference Engineering systems Design and Analysis (ESDA '04), pp. 65-72, Manchester, UK, July 2004.

[3] P. Singh and F. Nestmann, "An optimization routine on a prediction and selection model for the turbine operation of centrifugal pumps," Experimental Thermal and Fluid Science, vol. 34, no. 2, pp. 152-164, 2010.

[4] S. Derakhshan and A. Nourbakhsh, "Experimental study of characteristic curves of centrifugal pumps working as turbines in different specific speeds," Experimental Thermal and Fluid Science, vol. 32, no. 3, pp. 800-807, 2008.

[5] S. Derakhshan, B. Mohammadi, and A. Nourbakhsh, "Efficiency improvement of centrifugal reverse pumps," Journal of
Fluids Engineering, Transactions of the ASME, vol. 131, no. 2, Article ID 021103, 9 pages, 2009.

[6] S. Derakhshan, B. Mohammadi, and A. Nourbakhsh, "The comparison of incomplete sensitivities and Genetic algorithms applications in 3D radial turbomachinery blade optimization," Computers and Fluids, vol. 39, no. 10, pp. 2022-2029, 2010.

[7] P. Singh, Optimization of internal hydraulics and of system design for pumps as turbines with field implementation and evaluation, Ph.D. Thesis, Karlsruhe University, 2005.

[8] P. Singh and F. Nestmann, "Internal hydraulic analysis of impeller rounding in centrifugal pumps as turbines," Experimental Thermal and Fluid Science, vol. 35, no. 1, pp. 121-134, 2011.

[9] M. Harano, K. Tani, and S. Nomoto, "Practical application of high-performance francis-turbine runner fitted with splitter blades at ontake and shinkurobegawa no. 3 power stations of the Kansai electric power CO., Inc," Hitachi Review, vol. 55, no. 3, pp. 109-113, 2006.

[10] G. Mustafa, P. Yasar, and S. Yakup, "Energy saving in a deep well pump with splitter blade," Energy Conversion and Management, vol. 47, no. 5, pp. 638-651, 2006.

[11] J. P. Solano, V. Pinilla, G. Paniagua, S. Lavagnoli, and T. Yasa, "Aero-thermal investigation of a multi-splitter axial turbine," International Journal of Heat and Fluid Flow, vol. 32, no. 5, pp. 1036-1046, 2011.

[12] R. Barrio, J. Parrondo, and E. Blanco, "Numerical analysis of the unsteady flow in the near-tongue region in a volute-type centrifugal pump for different operating points," Computers and Fluids, vol. 39, no. 5, pp. 859-870, 2010.

[13] K. A. Kaupert and T. Staubli, "The unsteady pressure field in a high specific speed centrifugal pump impeller-part I: influence of the volute," Journal of Fluids Engineering, Transactions of the ASME, vol. 121, no. 3, pp. 621-626, 1999.

[14] R. Spence and T. Purdom, "Numerical prediction of transient loadings on multistage pump impellers," in Advances of CFD in Fluid Machinery Design, IMechE, London, UK, 2003.

[15] L. K. Stanmore, "Field problems relating to high-energy centrifugal pumps operating at part-load," in IMechE Conference C343/88 Pumps in Part Load, 1988.

[16] H. Keck and M. Sick, "Thirty years of numerical flow simulation in hydraulic turbomachines," Acta Mechanica, vol. 201, no. 1-4, pp. 211-229, 2008.

[17] S. Yang and F. Kong, "Numerical simulation and comparison of pump and pump as turbine," in ASME, Fluids Engineering Summer Meeting, pp. 1-10, Montreal, Canada, 2010.

[18] S. Carlos, M. Jesus, and M. Katia, "Numerical modelling and flow analysis of a centrifugal pump running as a turbine: unsteady flow structures and its effects on the global performance," International Journal for Numerical Methods in Fluids, vol. 65, no. 5, pp. 542-562, 2011.

[19] Help Navigator, ANSYS CFX, Release 12.0 CFX-Solver modeling Guide.

[20] S. Derakhshan and A. Nourbakhsh, "Theoretical, numerical and experimental investigation of centrifugal pumps in reverse operation," Experimental Thermal and Fluid Science, vol. 32, no. 8, pp. 1620-1627, 2008. 

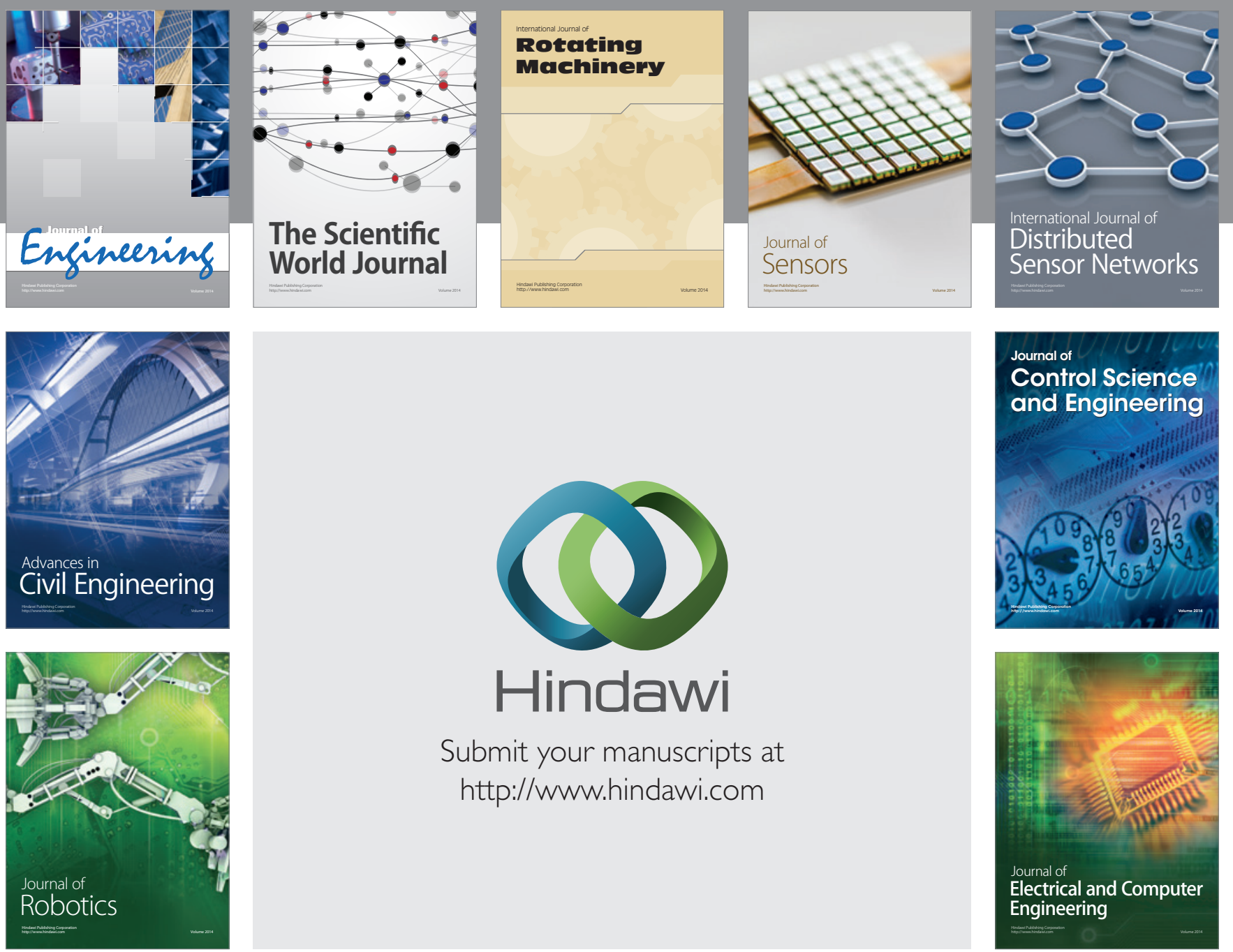

Submit your manuscripts at

http://www.hindawi.com
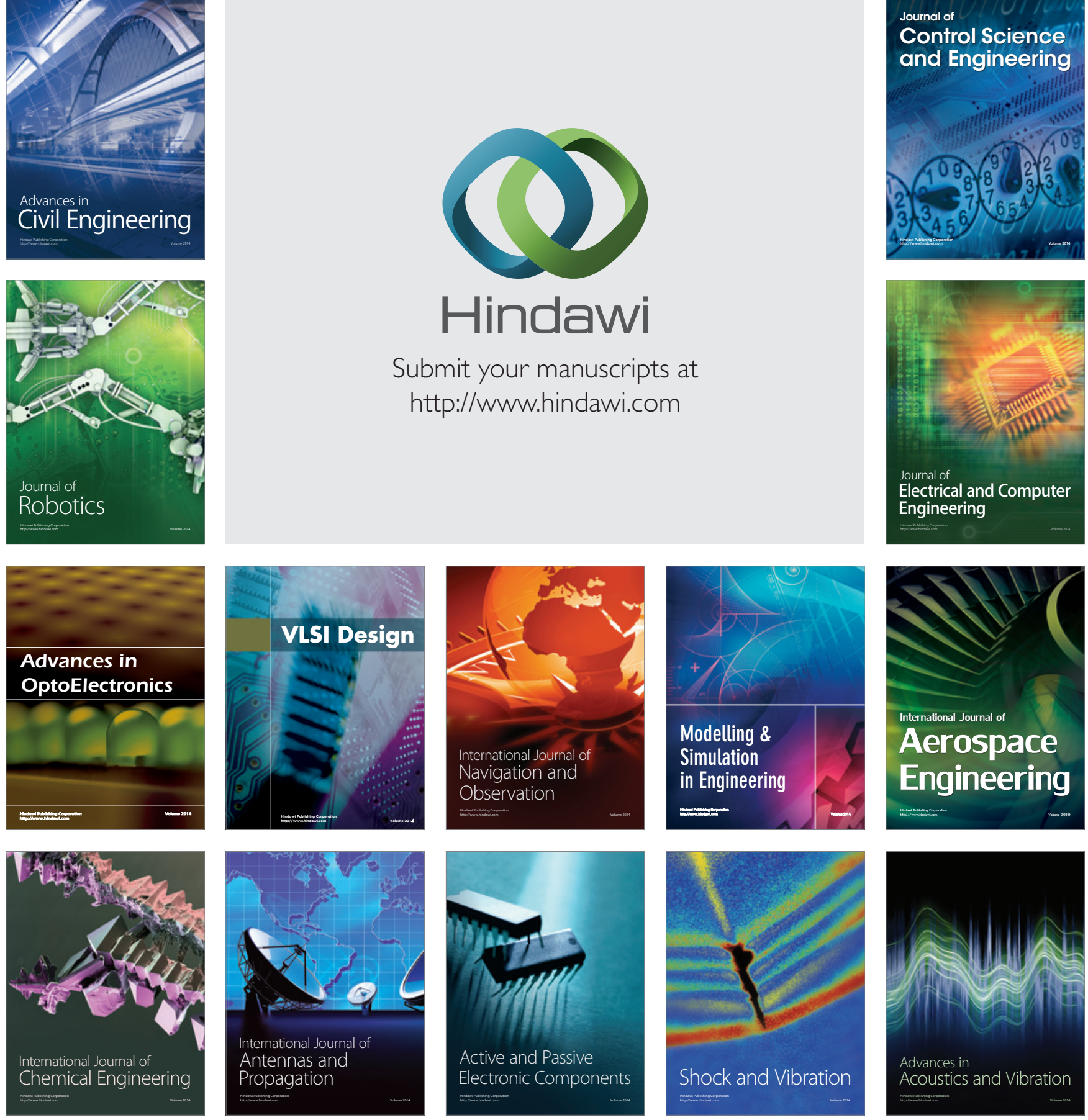